\title{
Zytologische Untersuchungen der menschlichen Prostataepithelien mit besonderer Berüicksichtigung der apokrinen Sekretion.
}

\author{
Von
}

Mineyosi Matusita.

Aus dem Anatomischen Institut der Keio Universität.

Mit ${ }_{4}$ Figuren auf Tafel X-XI.

Inhalt.

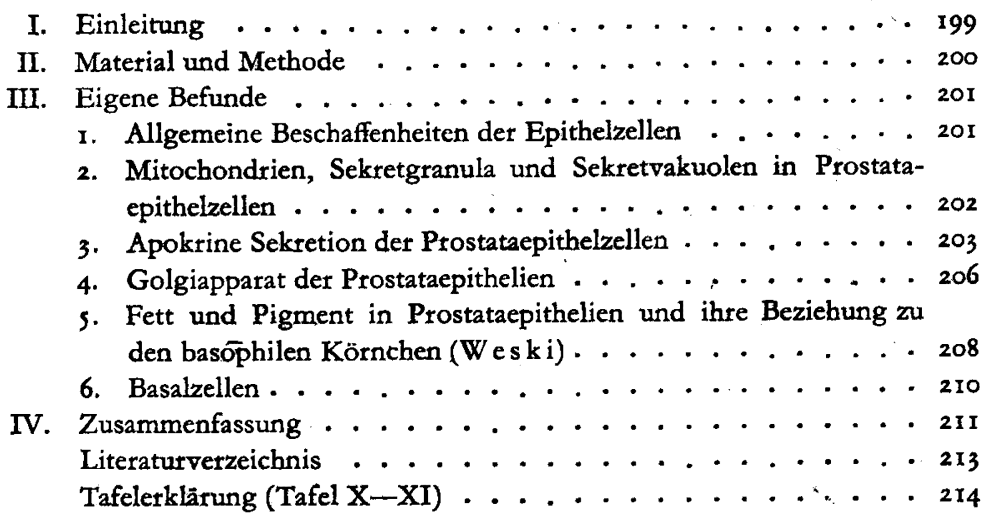

\section{Einleitung.}

Die Vorsteherdrüse stellt eine wichtige akzessorische Geschlechtsdrüse dar, und ist bis jetzt von zahlreichen Forschern zytologisch und histologisch studiert worden. In den Drüsenzellen der Prostata sind verschiedene Zytoplasmaeinschlüsse, wie Golgiapparat, Mitcchcndrien, S $€$ kretgranula, basophile sowie acidophile Körnchen, Pigmentgranula, Lipoidkörnchen u.a., bekantnt, aber es ist heute noch nicht endgültig gelöst worden, wieweit diese Einschlüsse sich an der Sekretion beteiligen. Die Bedeutung der basophilen und acidophilen Körnchen, welche von Weski (1903) und Petersen (1909) zuerst in den Drüsenzellen der menschlichen Prostata beschrieben wurden, ist heute noch nicht zufriedenstellend erklärt; die Beziehung zwischen diesen Körn- 
chen einerseits und Sekretgranula, Pigment- und Lipoidkörnchen anderseits muss man weiter eingehend erforschen. Der Golgiapparat der Drüsenzellen ist bei der menschlichen sowie tierischen Prostata von einer Anzahl Autoren nachgewiesen worden, doch ist Seine Veränderung in verschiedenen Sekretionsstadien kaum untersucht worden. Die Mitochondrien der Prostatadrüsenzellen wurden neuerdings von Takeichi (193I) bei Kaninchen eingehend beschrieben, bei Menschen sind sie bis heute, die kurze Abhandlung von Dominici ( 2913 ) ausgenommen, kaum studiert worden.

Über die Sekretionsweise der Prostatadrüsenzellen wurde von verschiedenen Autoren auf eine Besonderheit aufmerksam gemacht; Bonis (1907) und Eberth haben schon den unregelmässig zerrissenen, freien Rand der Drüsenzellen bemerkt. Nach der Angabe von Petersen (1909) und Stieve (1930) senden die Drüsenzellen der menschlichen Prostata oft unregelmässig geformte Protoplasmafortsätze ins Drüsenlumen aus. Diese Befunde deuten, nach meiner Ansicht, auf die apokrine Sekretion dieser Drüsenzellen hin, obwohl die oben genannten Forscher darüber nichts erwähnt haben. Erst neuerdings hat Röhlich (1938) die typische apokrine Sekretion der Prostatadrüsenzellen bei Menschen, Katze und Ratte beschrieben.

In dieser Untersuchung habe ich bei den ganz frischen menschlichen Vorsteherdrüsen die Drüsenzellen zytologisch eingehend beobachtet, um hauptsächlich die Veränderungen der Mitochondrien, Sekretgranula und des Golgiapparates bei verschiedenen Sekretionsstadien und die Beziehung zwischen Pigmentgranula, Lipoidkörnchen und sog. basophilen Körnchen von Weski genau zu studieren. Besonders konnte ich die apokrine Sekretion der menschlichen Prostatadrüsenzellen stufenweise eingehend beobachten.

\section{Material und Methode.}

Die Untersuchungsmaterial besteht aus menschlichen Vorsteherdrüsen, welche von ganz gesunden Hingerichteten ( 32 u. 33 jährig) im lebendfrischen Zustand herạusgenommen und fixiert wurden. Zum Zweck der Darstellung des Golgiapparates wurde die Kolatchevsche Osmiumimprägnationsmethode angewandt. Für die Darstellung der Mitochondrien habe ich mit Levischer Fixierungsflüssigkeit gute Resultate gewonnen. Die Lipoidkörnchen wurden bei oben genannten Methoden mit Osmiumsäure schwarz imprägniert dargelegt. Für die Herstellung der Übersichtspräparate wurden

Regaudsches Verfahren und Formol-Alkohol benutzt. Alle fixierten Gewebestücke kamen durch aufsteigende Alkoholreihe in Xylol und wurden in Paraffin eingebettet. Die nach Kolatchev behandelten Schnitte wurden 
mit Anilinfuchsin nach Kull gefärbt oder ohne Färbung beobachtet. Die Levi-Schnitte wurden mit Eisenhämatoxylin nach Heidenhain und die Übersichtspräparate mit Hämatoxylin(Hansen)-Eosin gefärbt. In den mit Regaudschem Verfahren fixierten Schnitten gelang es mir nicht, die Mitochondrien prächtig anzufärben.

Hier spreche ich Herrn Dr. G. Takahashi† und Herrn Dr. K. Kiso des Mantetu-Hospital zu Hsin-King für ihre freundliche Beihilfe bei Ansammlung des Untersuchungsmaterials, meinen herzlichsten Dank aus.

\section{Eigene Befunde.}

\section{Allgemeine Beschaffenheiten der Epithelzellen.}

Die Form der Epithelzellen ist sehr -verschieden; sie sind bald niedrig kubisch, bald hoch zylindrisch, Zwischenformen zwischen diesen beiden Formen aufweisend. Ganz platte Epithelzellen habe ich in dieser Untersuchung kaum getroffen. Diese hochgradige Schwankung der Zellformen beruht wenigstens zum Teil auf den verschiedenen Funktionszuständen der Epithelzellen, darüber werde ich unten wieder Erwähnung tun. Die Form des Kerns ist rundlich oder elliptisch, in hoch zylindrischen Epithelzellen ist sie häufig elliptisch. Der Kern kommt gewöhnlich einfach vor, selten trifft man zweikernige Zellen an. Bei den kubischen und zylindrischen Zellen befindet sich der Kern im allgemeinen mehr basal. Der Kern der Epithelzellen der Vorsteherdrüse sieht gewöhnlich dunkel aus, das Karyoplasma ist fein granulär. Das Kernkörperchen ist im allgemeinen klein, kommt ein- bis dreifach vor; die Lage des Kernkörperchens ist unbestimmt, so liegt es oft auf der Kernmembran.

Das Zytoplasma der kurz zylindrischen und zylindrischen Epithelzellen sieht meistens fein schaumig oder alveolär aus, wie von Dis selhor st (1904), Eberth (1904), Bonis (1907), Stieve (1930), Röhlich (1938) u. a. angegeben. Dies ist auf die Anhäufung der schwer färbbaren Sekrettröpfchen zurückzuführen. Das'Zytoplasma der niedrig kubischen und kubischen Zellen ist meistens ganz homogen gebaut.

Zwischen den einzelnen Epithelzellen findet man immer die Schlussleiste, welche sich bei senkrechtem Schnitte der Epithelzellen als mit Eisenhämatoxylin stark gefärbte Stäbchen erweist.

Zwischen den Epithelzeller und der Membrana propria findet man Basalzellen, welche, wie unten eingehend erwähnt, verschiedene Gestalt und Färbbarkeit zeigen. Die Basalzellen bilden aber nicht immer eine kontinuierliche Schicht, sie sind in mehreren Stellen unterbrochen. 


\section{Mitochondrien, Sekretgranula und Sekretvakuolen in Prostataepithelzellen.}

Wie oben kurz erwähnt, sieht das Zytoplasma der niedrig zylindrischen und zylindrischen Epithelzellen fein schaumig aus, nämlich diese Zellen enthalten kleine schwer färbbare Sekrettröpfchen(-vakuolen). Nur bei den niedrig kubischen und kubischen Zellen ist das Zytoplasma ganz homogen gebaut, enthält keine Vakuolen, somit hält man solche Zellen füł leere Żellen. Diese Zellen führen stäbchenförmige Mitochondrien und kleine färbbare Granula in kleiner Zahl, welche zum grössten Teil in der Umgebung des Kerns verteilt sind. Bei solchen niedrigen Zellen finden wir immer am freien Rand der Zelle eine mitochondrien- und granulafreie Zone (Fig. r). Bei etwas höheren Zellen, nämlich bei niedrigen Zylinderzellen tritt schon oberhalb des Kerns eine kleine Anhäufung der hellen Vakuolen auf (Fig. 2). Die stäbchenförmigen Mitochondrien sind in der Umgebung des Kerns und in dem Basalteil der Zelle zu finden. Zwischen den Vakuolen finden sich auch stäbchenförmïge Mitochondrien und kleine färbbare Granula. Am freien Rand der Zelle finden wir ebenfalls eine homogene, etwas dunkel erscheinende Plasmazone, welche weder Mitochondrien noch Granula führt. Diese riedrigen Zylinderzellen enthalten somit schon Sekretmasse, somit findet in diesen Zellen die Sekretbildung statt. Bei den in der Fig. 3 abgebildeten niedrigen Zylinderzellen sind die hellen Vakuolen in hohem Masse zugenommen und $z$ wischen diesen befinden sich verhältnismässig zahlreiche färbbare Granula und geringe Mitochondrien. Die Vakuolen füllen fast vollkommen den apikalen Teil der Zellen aus, doch bleibt die oben genannte dunkle, homogene Plasmazone erhalten. Oberhalb dieser dunklen Randzone liegt eine dünne helle Schicht, welche hügelartig in das Drüsenlumen vorwölbt.

Bei hoch zylindrischen Zellen ist das Zytoplasma von den zahlreichen kleinen Vakuolen ausgefüllt und bei Hochstand des-Kems treten unterhalb des Kerns, d.h. im Basalteil der Zelle auch helle Vakuolen auf (Fig. 4). Also sieht das Zytoplasma der hoch zylindrischen Zellen meistenfalls hell und fein schaumig aus, wie von verschiedenen Autoren angegeben ist, was ohne Zweifel infolge der Ansammlung der unfärbbaren Sekretmasse hervorgerufen ist. Bei solchen hoch zylindrischen Zellen findet man ebenso eine dunkle Plasmazone an der freien Oberfläche auf; oberhalb dieser findet sich bei den in der Fig. 4 abgebildeten Zellen ebenso eine helle Zone, welche nach dem Drüsenlumen hügelartig hervorragt. Zwischen den Vakuolen sind Mitochondrien und kleine färbbare Granula vorhanden. Unter den stäbchenförmigen Mitochondrien findet man häufig solche, an welchen die beiden Enden kuglich verdickt 
sind. Solche Mitochondrien stellen also Hantelform oder Doppelkörnchen dar, welche bei Abtrennung höchstwahrscheinlich je zwei Granula liefern. In dieser Weise entstehen scheinbar die färbbaren Granula von den Mitochondrien. Diese von Mitochondrien abstammenden kleinen färbbaren Granula entsprechen nach meiner Ansicht den kleinen acidophilen Körnchen der Autoren (Petersen (1909), Stieve (1930), Röhliçh (1938) u.a.), sie sind nichts anders als die Sekretgranula der Prostataepithelzellen. Sie wachsen nicht erheblich, so dass ihre Grösse nicht so weit variiert. Ihre Lage in der Zelle ist, wie in der Fig. 3, 4 u.a. abgebildet; unbestimmt. In seltenen Fällen habe ich in der Umgebung des Kerns der hochzylindrischen Zellen eine dichte Anhäufung der kleinen Granula gefunden; in solchen Zellen sind ausserdem keine stäbchenförmige Mitockondrien vorhanden, daher scheinen diese Granula zum Teil granuläre Mitochondrien, zum Teil Sekretgranula zu sein (Fig. 8).

Dominici (1913) hat in den Prostataepithelzellen des Menschen und Hundes die Mitochondrien und Sekretgranula nachgewiesen, doch hat er zwischen beiden Gebilden keine genetische Beziehung gefunden. Moor, Price und Gallagher (1930) haben in den Prostatazellen der Ratte zahlreiche Mitochondrien gefunden, aber sie haben über Sekretgranula keine Beschreibung vorgenommen. Takeichi (193x) hat bei den Prostatazellen des Kaninghens die genetische Beziehung zwischen Mitochondrien und Sekretgranula erkannt und festgestellt, dass die Sekretgranula von den Mitochondrien stammen, wie ich eben bei den menschlichen Prostatazellen bemerkt habe. Er hat dabei weiter erkannt, dass die Sekretgranula aufquellen, sich verfüssigen und in Sekretvakuolen umwandeln.

Ich konnte bei den menschlichen Prostatazellen diese Tatsache nicht bestätigen. So konnte ich die Befunde nicht finden, welche wirklich für die Umwandlung der kleinen färbbaren Granula in die hellen Vakuolen sprechen. Wenn auch es mikroskopisch unbemerkbar ist, findet der Vorgang wahrscheinlich auch bei menschlichen Prostataepithelien statt, dass die Sekretgranula sich verflüssigèn und in die unfärbbaren Vakuolen umwandeln. Also sei es berechtigt, dass man die oben erwähnten kleinen hellen Vakuolen in menschlichen Prostataepithelien als Sekretvakuolen bezeichnet.

\section{Apokrine Sekretion der Prostataepithelzellen.}

Aus obigen Befunden kann man wohl sagen, dass die niedrigen Epithelzellen det Prostata durch Ansammlung der Sekretvakuolen im Zytoplasma nach und nach zu höheren zylindrischen Zellen heranwachsen, deren freie Ober- 
fläche nach dem Drüsenlumen hügelartig vorwölbt. Bei solchen stark gefüllten Zylinderzellen springt dann die vorgewölbte freie Zellobe1fläche als kuppelförmiger Fortsatz weit in das Drüsenlumen vor (Fig. $5,6,7$ ), welcher mit den kleinen hellen Vakuolen ausgefüllt ist und ganz hell und fein schaumig erscheint, wie das eigentliche Zytoplasma selbst: Dieser Zytoplasmafortsatz ist immer von einer dünnen, schwach färbbaren Membran umhüllt. Bei solchen den kuppelförmigen Zytoplasmafortsatz führenden Zylinderzellen ist zwischen dem eigentlichen Zytoplasma und dem Fortsatz eine dunkle Zone vorhanden, welche zweifelsohne mit der dunklen homogenen Plasmazone der freien Oberfläche der niedrigen fortsatzlosen Zellen identisch ist. Diese dunkle Zone ist nun durch die hellen Sekretvakuolen mehrfach durchsetzt (Fig. $5,6,7$ ). So darf man bezüglich der Entstehung der kuppelförmigen Zytoplasmafortsätze folgendermassen denken, dass die im eigentlichen Zytoplasma gebildeten Sekretvakuolen diese dunkle Plasmazone hindurch in den Fortsatz übergehen, um dort nach und nach anzuhäufen. Die bei den in der Fig. 3, 4 abgebildeten Zellen bemerkte, helle hügelartige Zone oberhalb der betreffenden dunklen Plasmazone ist nichts anders als das erste Erscheinen des Zytoplasmafortsatzes. Die genannte dunkle Plasmazone ist in allen Sekretionsstadien immer erhalten und bildet die Scheidewand zwischen dem eigentlichen Zytoplasma und Sekretfortsatz. Die Grenze $z$ wischen der dunklen Plasmazone und dem hellen Sekretfortsatz ist immer verhältnismässig scharf, während diese Zone allmählich ins schaumige helle eigentliche Zytoplasma übergeht. Min a mi tani (1941) hat bei den Drüsenzellen der typischen apokrinen Schweissdrüse der menschlichen Achselhaut die gleiche dunkle und homogene Zytoplasmazone im superficialen Abschnitt der Zelle bemerkt und erkannt, dass diese Zone im ganzen Verlauf der apokrinen Sekretion eine wichtige Rolle spielt. Seine Beschreibung über-diese Zytoplasmazone stimmt mit den oben erwähnten gut überein. Diese Zytoplasmazone scheint für die apokrine Sekretion charakteristisch zu sein.

Der Inhalt der Sekretfortsätze besteht in der Regel ausschliesslich aus Sekretvakuolen, welche von dem eigentlichen Zytoplasma die dunkle Zytoplasmazone hindurch ins Innere der Sekretfortsätze herauswandert und ganz gleich beschaffen wie die Sekretvakuolen im Zytoplasma sind. Bei dem gewöhnlichen Sekretionszustand der Prostata enthält der Sekretfortsatz in der Regel weder Sekretgranula noch Mitochondrien. Der Sekretfortsatz ist somit ein Bläschen, welches flüssiges Sekretmaterial enthält.

Die in dieser Weise gebildeten Sekretfortsätze wachsen mit der Zunahme der Sekretvakuolen ins Drüsenlumen allmählich heran (Fig. 5, 6, 7), Dann wird der Wurzelteil des Fortsatzes stielartig abgeschnürt und schliesslich 
trennt sich der Fortsatz im abgeschnürten Teil ins Drüsenlumen als tropfenartige Sekretmasse ab (Fig 7), oder bei den anderen Fällen bricht die dünne Oberflächenmembran des Sekretfortsatzes in einer Stelle auf und der Inhalt wird ins Drüsenlumen entleert. Bei in diesen Weisen den Sekret abgeschiedenen Zellen bildet die genannte dunkle Zytoplasmazone nun wieder die freie Zelloberfläche.

Wenn man den eben geschilderten Sekretiosmodus der Prostataepithelzellen anblickt, so versteht man ohne weiteres, dass diese Zellen eine typische apokrine Sekretion durchmachen. Von früheren Zeiten wurde es von einigen Autoren aufmerksam gemacht, dass die Epithelzellen der Prostata eine von der gewöhnlichen ekkrinen Sekretion mehr oder weniger abweichende Sekretion zeigen. So hat Eberth (1904) beschrieben, dass die Kontur der Zellen bald scharf, bald hervorgewölbt, unregelmässig zerrissen ist, und dass, im letzteren Falle das Sekret direkt mit dem Inhalt der Zelle in Berührung steht. Bonis (1907) hat bei der Prostata des Hundes erkannt, dass der freie Rand der mit fuchsinophilen Körnchen und Plasmosomen gefüllten Zellerr aufbricht und die Körnchen ins Drüsenlumen entleert worden. Petersen (I909) hat in der mikroskopischen Untersuchung der menschlichen Prostata beschrieben, dass die mit acidophilen Körnchen gefüllten Epithelzellen oft die unregelmässig geformten Protoplasmaausläufer ins Drüsenlumen aussenden, deren periphere Partien durch Abschnürung ins Drüsenlumen als unregelmässig geformte Sekretmassen abgetrennt werden. 'So hat Petersen schon mit Sicherheit die apokrine Sekretion der Prostataepithelien nachgewiesen. Der Terminis der ,apokrinen Sekretion“ wurde zuerst im Jahre 1922 von Schiefferdecker in seiner berühmten Untersuchung der Hautdrüsen in die Morphologie eingeführt, so ist es freilich, dass Petersen diese Bezeichnung nicht angewandt hat. Stieve (1930) hat ir seinem Handbuchbeitrag bei den menschlichen Prostataepithelien die längeren oder kürzeren Fortsätze, welche in den Drüsenhohltaum vorspringen und sich in ihm verlieren, beschrieben, doch hat er die Bezeichnung apokriner Sekretion nicht benutzt. Neuerdings hat Röhlich (1938) bei den Prostataepithelien des Menschen und der Katze sowie der Ratte die gleichen Befunde wie Petersen und Stieve bemerkt und zum ersten Mal die Sekretionsweise dieser Epithelien als typische apokrine Sekretion bezeichnet. Ich konnte in der vorliegenden Untersuchung der menschlichen Prostataepithelien die Angabe von Röhlich einwandfrei bestätigen.

Wie von Forschern aufmerksam gemacht, schwankt die Form der Prostataepithelien in hohem Masse. Petersen (1909) hat dafür drei Faktoren angegeben, welche die Zellform der Prostataepithelien bestimmen: den 
Druck des in Drüsenlumen angehäuften Sekretes, den Füllungszustand der Zelle und den Kontraktionszustand der Muskulatur im interstitiellen Bindegewebe. Röhlich (1938) gibt ausser dem Kontraktionszustand der glatten Muskulatur die apokrine Sekretion der Prostataepithelien an. Nach seiner Meinung ist das Niedrigwerden der Zylinderzellen die Folge der mehrmals nacheinander stattfindenden apokrinen Sekretion.

Ich schliesse mich auch der Ansicht von Röhlich an; infolge der apokrinen Sekretion geht das Zytoplasma zum Teil verloren, so bei mehrmals nacheinander folgender apokriner Sekretion werden die Zylinderzellen allmählich niedrig. Die starke Schwankung der Zellform wird auch bei den Epithelzellen der apokrinen Schweissdrüse gefunden. Doch sollen die Einflüsse des Druckes des Sekretes im Drüsenlumen und der Kontraktion der glatten Muskulatur nicht gänglich ausgeschlossen werden. Ich habe in den menschlichen Prostataepithelien häufig bemerkt, dass die niedrig zylindrischen und kubischen Epithelzellen die Sekretfortsätze ins Drüsenlumen aussenden, wie in der Fig. 9 wiedergegeben. Dieser Befund weist hin, dass die wahrscheinlich infolge mehrmaligen apokrinen Sekretionen niedrig gewordenen Epithelzellen noch die apokrine Sekretion wiederholen.

Die durch mehrmalige nacheinander folgende apokrine Sekretionen ganz erschöpften Zellen fallen wahrscheinlich erst in Ruhezustand, solche Zelleñ sind durch zuerst beschriebene 'niedrige Epithelzellen vertreten, welche stäbchenförmige Mitochondrien und geringe kleine färbbare Granula, aber keine Vakuolen enthalten (Fig. r).

\section{Golgiapparat der Prostataepithelien.}

Der Golgiapparat der Prostataepithelien wurde zuerst von Berg en (1904) bei Hund beschrieben. Neuerdings wurde er bei Ratte, Meerschweinchen und Kaninchen von Moor, Price und Gallagher (1930) Turchini und Bronssy (1930) und Takeichi (1931) nachgewiesen. Bei Menschen wurde er auch von Verson (1908), Taddei (1910) und Kopsch (1925) beobachtet. Verson und Taddei haben die durch chirurgische Operation entnommenen Drüsen untersucht. Nach den Befunden dieser beiden Autoren ist der Golgiapparat der Prostataepithelzellen unmittelbar oberhalb des Kerns angelagert und besteht in der Mehrzahl der Fälle nur aus einem gewundenen Faden, welcher oft Ring, Schlinge, kleine Knoten bildet. Kopsch hat bei der von zwei Hingerichteten herausgenommenen ganz frischen Prostata den Golgiapparat mittels Osmiumimprägnationsmethode dargestellt. Nach seiner Beschreibung liegt der Golgiapparat der Prostatazellen als geschlossenes System oberhalb des Kerns und zwar näher oder weiter von ihm. In 
den abgeplatteten Epithelzellen liegt der Golgiapparat ganz oder zum Teil seitlich an dem abgeplatteten Kern. Die oben genannten Autoren haben aber bei der Beobachtung des Golgiapparates die Funktionszustände der Zelle nicht berücksichtigt.

Nach meinen eigenen Befunden besteht der Golgiapparat der menschlichen Prostataepithelien aus den mit der Osmiumsäure schwarz imprägnierten, gewundenen Strängen. Im Gegensatz zu den Befunden von Kopsch, Takeichi u.a. bilden die Stränge kaum das komplizierte Netzwerk; in dieser Hinsicht stimmt mein Befund mit dem von Verson überein.

In den mehr abgeplatteten Epithelzellen, welche noch keine Sekretvakuolen enthalten, liegt der Golgiapparat am häufigsten seitlich an dem Kern, wo das Zytoplasma am reichlichsten vorhanden ist (Fig 10). So habe ich die Befunde von Kopsch bestätigt. Bei den mehr abgeplatteten oder niedrig kubischen Zellen kann der Golgiapparat aber unmittelbar oberhalb des Kerns vorkommen, falls der Zytoplasmaraum dort verhältnismässig weit ist.

Tritt die Sekretbildung ein, so treten im Zytoplasma Sekretvakuolen auf; mit der Ansammlung der letzteren nimmt die Höhe der Zelle zu. Bei solchen Zellen liegt der Golgiapparat immer oberhalb des Kerns. In den der Fig. II abgebildeten niedrig zylindrischen Zellen liegt der einfach gebaute Golgiapparat oberhalb des Kerns, die dort angesammelten Sekretvakuolen sind innerhalb des Golgiapparates eingeschlossen. Dabei stellt der Apparat oft ein die Sekretvakuolen enthaltendes geschlossenes System dar. Bei den hochzylindrișchen, grösseren oder kleineren Sekretfortsatz führenden, mit den Sekretvakuolen gefüllten Zellen vergrössert und dehnt sich der Golgiapparat mehr nach apikal aus, indem er die unmittelbar oberhalb des Kerns befindlichen hellen Sekretvakuolen umschliesst (Fig. 12). Der Bau des Golgiapparates ist gleich wie bei den oben erwähnten niedrigeren Zellen; er stellt ein einfaches Netzwerk dar.

Bei diesen hochzylindrischen Zellen findet man oberhalb des Golgiapparates auch Sekretvakuolen, so scheinen diese von dem Innern des Golgiapparates nach apikalwärts abgeschickt $z u$ werden, um dann weiter die dunkle homogene Zytoplasmazone hindurch in Zytoplasmafortsatz ausgeschieden zu werden, welche durch Osmiumimprägnation besonders dunkel angefärbt wird.

Aus obigen Befunden kommt man zu der Vermutung, dass zwischen dem Golgiapparat und den Sekretvakuolen eine innige Beziehung bestehe; die Sekretvakuolen erscheinen zuerst innerhalb des Golgiapparates, dort werden sie wahrscheinlich gebildet und nach der Reifung werden sie erst 
in den Sekrètfortsatz ausgeschieden.

Wir finden auch die Sekretgranula innerhalb des Golgiapparates, sie werden bei $\mathrm{Kullscher} \mathrm{Färbung} \mathrm{der} \mathrm{Kolatchev-Präparate} \mathrm{mit} \mathrm{Fuchsin}$ gefärbt.

Beim Überblick des Golgiapparates der Prostataepithelien erkennt man die Tatsache, dass Form und Bau des Golgiapparates sich nach den verschiedenen Sekretionsstadien kaum verändern. In dieser Hinsicht stimmen die Prostataepithelien auch mit den Epithelzellen der apokrinen Schweissdrüse der menschlichen Achselhaut überein (Minamitani (I94I)).

\section{Fett und Pigment in Prostataepithelien und ihre Beziehung zu den basophilen Körnchen (Weski).}

Es wurde von zahlreichen Autoren beschrieben, dass die Prostatazellen physiologisch Lipoidkömchen enthalten. Über diese Frage hat Kinoshita (1920) eine sehr eingehende Untersuchung ausgeführt. Man kann bei ihm ein näheres Literaturverzeichnis finder. Nach seinen Ergebnissen treten Lipoidkörnchen in Prostatazellen des Menschen nach der Pubertätszeit konstant auf. Sie befinden sich hauptsächlich in der Zellbasis, d.h. unterhalb des Kerns. Nach seiner Meinung sind die Lipoidkörnchen nicht Sekretkörnchen, sondern Stoffwechselprodukt. Petersen (1909) und Kopsch (1925) haben bei Menschen, Moor, Price und Gallagher (1930) bei Ratte die Lipoidkömchen in Prostatazellen beschrieben. Diese Autoren haben alle festgestellt, dass die Lipoidkörnchen überhaupt im basalen Abschnitt der Zelle vorkommen.

Pigmentkörnchen wurden schon von Koelliker (1854) und Langerhans (1874) in Prostatazellen bemerkt. Eberth (1909) hat auch in der Umgebung des Kerns I-2 gelbliche Körnchen gefunden. Petersen (1909) hat dieselben in den Prostatazellen des Menschen nicht wahrgenommen. Nach Stieve (1930) ist auch die Pigmentablagerung bei gesunden jüngeren Leuten nicht zu finden, er hat aber bei älteren Leuten die Pigmentkömchen. im inneren Abschnitte der Zylinderzellen einzeln gefunden. Also sind die Pigmentkörnchen in Prostatazellen nicht so näher wie die Lipoidkörnchen untersucht worden.

Anderseits ist das Vorkommen der basophilen Körnchen in Prostatazellen seit Weski's Entdeckung von einigen Autoren aufmerksam gemacht worden. Weski (1903) hat zuerst bei Menschenprostata in der Umgebung des Kerns der Epithelzellen grosse basophile Körnchen gefunden und als , Sekretkugeln" bezeichnet, und meinte, dass sie nach der Ausscheidung zu dem Material der Prostatasteine werden. Die gleichen Bildungen wurden dann von Peters en 
(1909), Fischel und Kreibich (1911), Stieve (1930), Röhlich (1938) u.a. berichtet. Das Wesen der basophilen Körnchen ist ungeachtet der Untersuchungen von Autoren ganz unklar, also sind eine rätselhafte Bildung.

Ich habe in der vorliegenden Untersuchung bei den mit den Osmiumsäure enthaltenden Fixierungsgemischen behandelten Präparaten, Leviund Kolat che v-Präparaten, in dem Basalteil der Epithelzellen die Lipoidkörnchen häufig in kleiner Zahl nachgewiesen, welche mit Osmiumsäure schwarz tingiert werden (Fig. $x, 2,3,4,5,6,7,9$, ir, 12). Sie sind im allgemeinen grob, doch schwankt ihre Grösse in gewissem Masse. Innerhalb der grösseren Körnchen ist oft helle Vakuole enthalten. Bei Eisenhämatoxylinfärbung färben sich die Lipoidkörnchen häufig zum Teil; der färbbare Teil nimmt gewöhnlich die Rindenzone der Körnchen und stellt einen Ring oder Halbmond dar. Moor, Price und Gallagher (1930) haben auch bemerkt, dass die Lipoidkörnchen mit Fuchsin tingiert worden können.

Die Lipoidkörnchen kommen in der Regel im Basalteil der Zelle oder in der unmittelbaren Umgebung des Kerns vor.

Was die Genese der Lipoidkörnchen anbelangt, so scheint es mir wahrscheinlich zu sein, dass sie im Basalteil der Zelle von den kleinen färbbaren Granula, welche möglicherweise von Mitochondrien, ab stammten, entstehen. Solche Granula verlieren mit der Vergrösserung allmählich vom Zentralabschnitt ihre Färbbarkeit und zeigen Lipoidreaktion und wandeln in Lipoidkörnchen um.

Ich habe bei den mit Formol-Alkohol fixierten und mit HämatoxylinEosin gefärbten Präparaten in dem Basalabschnitt oder in der Umgebung des Kerns der Epithelzellen häufig grössere Körnchen in geringer Zahl vorgefunden (Fig. 14). Diese Kömchen enthalten oft Vakuolen wie die Lipoidkörnchen. Sie sind oft mit Hämatoxylin gefärbt, daher entsprechen sie den Weskischen basophilen Körnchen. Bei schwächerer Färbung mit Hämatoxylin erscheinen sie orange, bei gänzlich ungefärbten Fällen sehen sie hellgelb aus. Also kann man verstehen, dass die grösseren basophilen Körnchen gelbliche Eigenfarbe besitzen, und dass sie mit den Pigmentgranula von Autoren identisch sind.

Anderseits stimmen die basophilen Körnchen mit den Lipoidkörnchen, wie oben beschrieben, in ihrer Lage, Form und Grösse ganz überein. So , kam ich nun zu der-Annahme, dass die beiden die identischen Bildungen sein dürfen. Wenn die beiden die besondere Bildungen wären, so müssten wir in den mit Eisenhämatoxylin gefärbten L e vi-Präparaten die mit Osmiumsäure geschwärzten Lipoidkörnchen und die basophilen Körnchen als selbständige Bildungen gleichzeitig nachweisen. In Wirklichkeit ist dies nicht der Fall, denn wir nehmen in Levi-Präparaten nur die Lipoidkörnchen wahr. 
Ich möchte wie folgt schliessen : Die Prostatazellen besitzen im Basalabschnitt oder in der Umgebung des Kerns die gröbere Körnchen in geringer Zahl, welche hauptsächlich aus Eiweiss und Lipoid bestehen und gelbliche Eigenfarbe haben. Sie werden in den mit Osmiumgemisch fixierten Präparaten mit Osmiumsäure angeschwärzt und als Lipoidkörnchen wahrgenommen, aber sie erscheinen in den mit das Lipoid nicht konservierenden Fixierungsgemischen fixierten Präparaten als basophile Kömchen und bei ungefärbten Fällen als Pigmentkömchen. Also seien die von Autoren beschriebenen Lipoid-, basophile und Pigmentkörnchen meiner Ansicht nach die identischen Bildungen. Die von Autoren als Lipoidkömchen bezeichneten und chemisch (Policard und Noel (1920) u.a.) und physikalisch (Romeis (1926 u.a.) eingehend untersuchten Bildungen sind chemisch nicht einfach; sie enthalten scheinbar Eiweisssubstanzen. (Nach Stieve'(1930) gehören die basophilen Körnchen zu Eiweisssubstanzen).

Die physiologische Bedeutung dieser gröberen basalen Körnchen ist unklar. Nach Kinoshita (1920) sind die Lipoidkörnchen nicht Sekretkörnchen, sondern Stoffwechselprodukt." Nach Weski (1903) sind die basophilen Körnchen Sekretkugeln, nach Petersen (1907) bilden sich die basophilen Kömchen in acidophile Kömchen um, welch letztere endliche intracelluläre Sekretionsprodukte sind.

Nach meinen Ergebnissen stammen sowohl die Sekretgranula (acidophile Körnchen von Autoren) als auch die Lipoidkömchen gleich von den Mitochondrien $a b$, doćh gibt es keine Befunde, welche von der genetischen Wechselbeziehung der beiden Bildungen sprechen. Und es gibt auch keine Befunde, welche Ausscheidung der gröberen basalen Körnchen ins Drüsenlumen unterstützen.

\section{Basalzellen.}

Die Tatsache, dass unter dem einschichtig angeordneten Epithel der Prostata eine Schicht der Basalzellen vorhanden ist, welche hie und da unterbrochen ist, wurde von Eberth (1904), Petersen (1909), Stieve (193Q) Röhlich (1938) u.a. aufmerksam gemacht. Weski (1903) hielt diese Zellen irrtümlich für Bindegewebeszellen.

Nach meinèn eigenen Befunden sind die Form und Grösse dieser Zellen erheblich verschieden; bald sind sie ganz platt (Fig. 2), bald aber hoch (Fig. 6,12). Die Form des Kerns ist entsprechend der Gestalt der Zelle schwankend.

Die Mitochondrien sind schwer nachweisbar; in den ganz abgeplatteten Basalzellen konnte ich sie nicht vorfinden, in den höheren Zellen fand ich 
körnchenförmige und stäbchenförmige Mitochondrien in'geringer Zahl (Fig, 6. 8).

Der Golgiapparat der Basalzellen ist fast gleich wie derselbe der niedrigen Epithelzellen gebaut (Fig. 13). Er besteht aus gewundenen Strängen, welche nie kompliziertes Netz bilden, zuweilen nur aus kurzen Stäbchen und liegt bald oberhalb des Kerns, bald séitlich von demselben (Fig. 13).

Nach den zytologischen Befunden haben die Basalzellen kein Sekretionsvermögen; sie enthalten weder Sekretgranula noch Sekretvakuolen. Ich habe in dieser Untersuchung nur äusserst selten grössere kegelförmige Basalzelle gefunden, welche oberhalb des Kerns einige Sekretvakuolen enthält (Fig. 5). . Bei Auswachsen solcher Zelle entsteht höchstwahrscheinlich eine gewöhnliche sekretionsfähige Zylinderepithelzelle. Daher sind die Basalzellen die Ersatzzelle. Nach Röhlich (1938) sezernieren Basalzellen in der Epithelleiste, ihr Sekret sammelt sich.innerhalb der letztere und bildet die Cyste. Ich konnte in dieser Untersuchtung solche Erscheinung nicht bestätigen.

\section{Zusammenfassung.}

In der vorliegenden Untersüchung habe ich die Epithelzellen der im lebendfrischen Zustand entnommenen ménschlichen Prostata aus Hingerichteten zytologisch eingehend untersucht. Die wichtigen Ergebnisse werden im folgenden zusammenfassend angegeben.

I) Zum grössten Teil sind die Epithelzellen der menschlichen Vorsteherdrüse mit den kleinen hellen Sekretvakuolen gefüllt, so dass das Zytoplasma hell und schaumig aussieht. Nur die niedrigen Epithelzellen entbehren der Sekretvakuolen und das Zytoplasma ist homogen.

2) Die Mitochondrien sind stäbchenförmig und im ganzen Zytoplasma verstreut verteilt, indem sie auch zwischen den Sekretvakuolen gefunden werden.

3) Im allgemeinen sind die Sekretgranula gering und klein, sie wachsen nicht zu erheblicher Grösse. Die Sekretgranula kommen zwischen den Sekketvakuolen und in der Umgebung des Kerns. Sie stammen von den Mitochondrien ab und verwandeln sich höchstwahrscheinlich in Sekretvakuolen, obwohl wir in dieser Untersuchung diesen Vorgang morphologisch nicht verfolgen konnten. Die acidophilen Körnchen von bisherigen Autoren entsprechen nach meiner Meinung den Sekretgranula.

4) Der Golgiapparat dér Prostataepithelzellen stellt in der Regel - kein kompliziertes Netzwerk. Er kommt meistens oberhalb des Kerns vor. Die Sekretvakuolen treten zuerst innerhalb des Golgiapparates ; mit der Zunahme der ersteren dehnt der letztere sich gegen die freie Oberfläche der Epithel- 
zellen aus. Dann werden die Sekretvakuolen von dem Golgiapparat in,den Apikalteil der Zelle entsendet. Der Golgiapparat der Prostatazellen ändert sich aber nach den Funktionsstadien morphologisch kaum.

5). Die Prostataepithelien machen eine typische apokrine Sekretion durch. Die mit Sekretvakuolen gefüllten hochzylindrischen Zellen entsenden in das Drüsenlumen einen langen kuppelförmigen Fortsatz, welcher helle Sekretvakuolen, aber nìcht Sekretgranlua- enthält. Bald wird der Wurzelteil des Sekretfortsatzes stielartig abgeschnürt und trennt sich in dieser Stelle in das Drüsenlumen ab, bald bricht der Sekretfortsatz auf und wird der Inhalt desselben ins Drüsenlumen entleert. Infolge der wiederholten apokrinen Sekretion werden die Epithelzellen allmählich niedrig. Infolge der apokrinen Sekretion wetden wahrscheinlich verschiedene niedrige Formen der Prostataepithelien gebildet.

6) An der freien Oberfläche der Epithelzellen findet sich immer eine dunkle homogene Zone, wetche weder Mitochondrien noch Sekretgranula führt. Bei den Sekretfortsatz tragenden Epithelzellen bildet diese Zone die Scheidewand zwischen dem ersteren und dem eigentlichen Zytoplasma; die Sekretvakuolen gehen diese Zone hindurch ins Sekretfortsatz über und nach der Abtrennung oder Platzung des letzteren bildet diese Zone wieder die freie Oberfläche der Zelle. Diese dunkle homogene Zone scheint daher eine wichtige Rolle bei der apokrinen Sekretion zu spielen.

7) Im Basalteil der Epithelzellen kommen häufig gröbere Lipoidkörnchen vor, welche von den von Mitochondrien hergeleiteten färbbaren Granula abgestammt haben. Diese Lipoidkörnchen sind in den mit Formol-Alkohol fixierten Paraffinschnitten nicht vollkommen aufgelöst und stellen die mit Hämatoxylin mehr oder weniger färbbaren gröberen Körnchen dar. Dabei bemerkt man, dass sie eine gelbliche Eigenfarbe besitzen. Also kann man schliessen, dass die basophilen Körnchen (Weski), Pigment- und Lipoidkörnchen in Prostataepithelzellen die identischen Bildungen sind. Die Bedeutung dieser Bildungen ist unklar, aber es ist versichert, dass diese Bildungen nicht ins Drüsenlumen abgesondert werden.

8) Die sogenannten Basalzellen sind Ersatzzellen der sezernierenden Epithelzellen; sie stellen unterhalb der letzteren einschichtig angeordnete niedrige Zellenlage dar, welche hie und da unterbrochen ist. Der Golgiapparat bildet bald einfaches Netzwerk, bald besteht er nur aus kurzen Strängen und liegt oberhalb oder seitlich des Kerns. Die Mitochondrien werden in den platten Zellen kaum nachgewiesen, in den höheren als feine Körnchen oder Stäbchen in kleiner Zahl getroffen. Die Sekretgranula, Sekretvakuolen und Lipoidkömchen, sind gewöhnlich nicht enthalten. Die Basalzellen besitzen daher kein Sekretionsvermögen. 
Zum Schlusse spreche ich Herrn Prof. Dr. S. "Mochizuki für seine stete Unterstützung und Herrn Dozent Dr. T. Ito für seine liebenswürdige Leitung bei Ausführung dieser Arbeit meinen herzlichsten Dank aus.

\section{Literaturverzeichnis.}

Bergen, Fr. V., .rgo4. Zur* Kenntnis gewisser Strukturbilder im Protoplasma verschiedener Zellarten. Arch. mikr. Anat. Bd. 64. S. 498.

Bon is, V. de, 1907. Uber die Sekretionserscheinungen in den Drüsenzellen der Prostata. Arch. Anat. u. Entw.-gesch. 1907. S. I.

Disselhorst, I904. Ausführungsapparat und Anhangsdrüsen der männlichen Geschlechtsorgane. Jena. I904.

Dominici, M., 1913. Sulle formazioni mitocondriali e sui granuli di secrezione nella prostata del cane e nella prostata umana ipertrofica. Fol. Urolog. Bd. 7. S. 295.

Eb erth, K. v., I904. Die männlichen Geschlechtsorgane. Jena. I904.

Fischel, A. und Kreibich, Igrr. Uber Prostatasekretion. Wien. Klin. Wschr. IgrI،

Kinoshita, M., 1920. Die Lipoide der Prostata: Z. Urolog. Bd. 14. S: 145.

Koelliker, A., 1854. Mikroskopische Anatomie. II. Leipzig.

Kopsch, Fr., r926. Das Binnengerüst in den Zellen einiger Organe des Menschen. Z. mikranat. Forsch. Bd. 5. S. 221 .

Langerhans, P., 1874. Über die accessorischen Drusen der Geschlechtsorgane. Virchows Arch. Bd. sI.

Minamitani, K., I94I. Zytologische und histologische Untersuchungen der Schweissdrüsen in menschlicher Achselhaut. Zur Zytologie der apokrinen Schweissdrüsen in der menschlichen Achselhaiut. Fol. anat. jap. Bd. 21. S. 6x.

Moor, C. R., Price, D. und T. F. Gallagher., 1930. Rat prostate cytology as a testis hormone indicator and the prevention of castration changes by testis extract injections. Amer. Jour.' Anat. Vol. 45. P. 71.

Petersen, O.V.C.E., 1909. Beittãge zur Histologie der Prostata. Anat. Heft. Bd. 39. S. 653. Policard, A. und R. Noel, 1920. C. r. Soc. Biol. Paris. T. 83. P. 868. (Zit. nach Stie ve).

Röhlich, K., 1938. Über die Prostatasekretion. Z. mikr.-anat. Forsch. Bd. 43. S. 45 I.

Rome is, B., 1926. Hoden, samenleitende Organe und akzessorische Geschlechtsdrüsen. Handb. d. normalen u. pathologischen Physiol. Bd. 14. Heft r. Teil r. S. 693.

Schiefferdecker, r922. Die Hautdrüsen des Menschen und der Säugetiere, ihre biologische und rassenanatomische Bedeutung, sowie Muscularis sexualis. Zoolqgica. Heft. 72.

Stieve, H., I930. Männliche Genitalorgane. Möllendorffs Handb. d. mikr. Anat. d. Mensch. Bd. 7. Teil 2.

Taddei, C., r9ro. Sulla apparato reticolare interno di Golgi negli elementi epiteliali della prostata ipertrofica. Sperimentale Bd. 64. S. 434. (Zit. nach Kopsch).

Takeichi, T., r93 I. Zytologische Untersuchungen über die Epithelien der akzessorischen Geschlechtsdrüsen. IV. Mitt. Fukuoka Acta Medica. Bd. 24. S. 1329.

Takeichi, T., 193x. Zytologische Untersuchungen über die Epithelien der akzessorischen Geschlechtsdrüsen. V. Mitt. Fukuoka Acta Medica. Bd. 24. S. 1338.

Turchini u. Bronssy. 1930. (Zit. nach Moor, Price u. Gallagher).

Verson, S., xgo8. Contributo allo studio delle cellule giganti tubercolare e di altri element cellulari normali e patologici. Arch. Sci. med. Bd. 32. S. 489.

Weski, O., I903. Beiträge zur Kenntnis des mikroskopischen Baues der menschlichen Prostata. Anat. Heft. Bd. 21. S. 6r. 


\section{Tafelerklärung (Tafel X*XI)}

Alle Figuren sind mit dem A bbeschen Zeichenapparat auf der Objekttischhöhe gezeichnet und zwar mit 'Zeiss' Achromat-Imm. $\frac{1}{12}$, n.A. 1.25, Komp.-Ok. 12, in Tubuslänge $160 \mathrm{~mm}$.

Fig. 1-9. Fixiert mit Levi schem Osmiumgemisch, gefärbt mit Eisenhämatoxylin nach Heidenhain. Die Fettröpfchen sind hell schwarz gezeichnet.

Fig. 10-13. Behandelt mit Kolatchevscher Osmiumimprägnationsmethode, gefärbt mit Kull scher Anilinfuchsin-Aurantia-Methode. . Die Fettröpfchen sind auch bell schwarz gezeichnet.

Fig. 14. Fixiert mit Formol-Alkohol, gefärbt mit Hämatoxylin(Hans en)-Eosin. Basophile Körnchen (Weski) sind hellschwarz gezeichnet. 

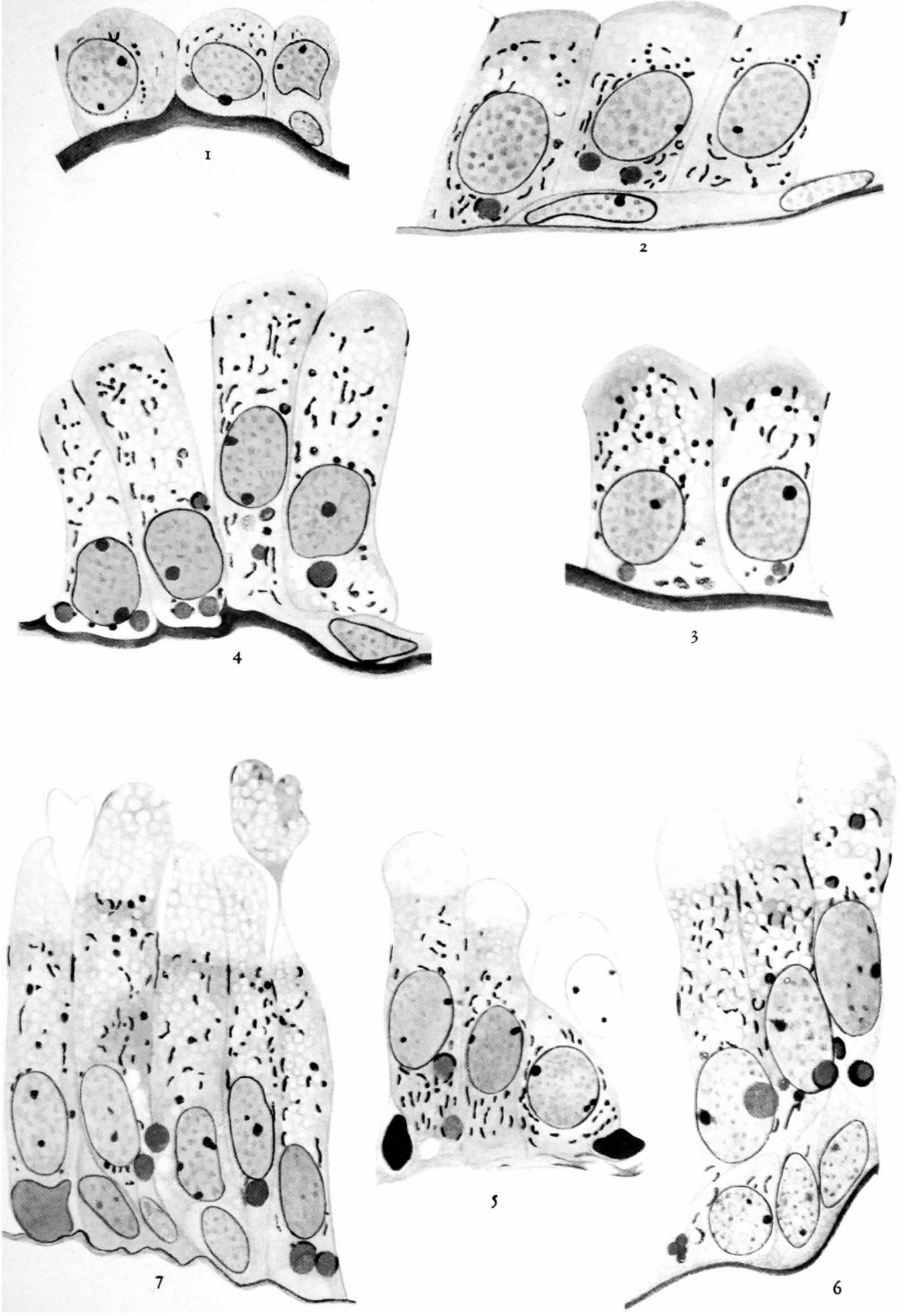

Matusita. 

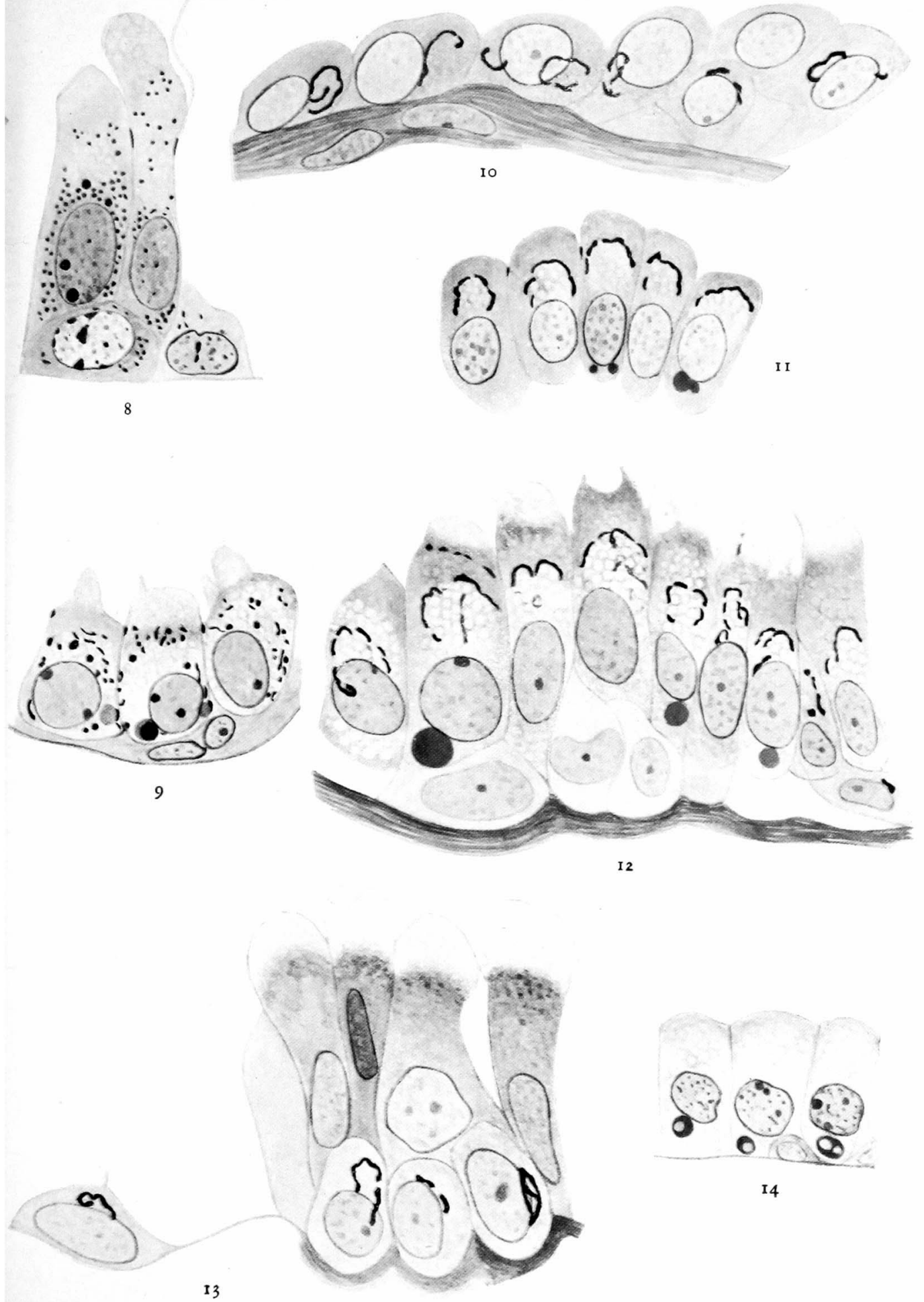

I4

Matusita. 